\title{
Chemical composition and biological activities of Juçara (Euterpe edulis Martius) fruit by-products, a promising underexploited source of high-added value compounds
}

\author{
Jéssica A.A. Garcia ${ }^{a, b}$, Rúbia C.G. Corrêa ${ }^{a, b, c}$, Lillian Barros ${ }^{c}$, Carla Pereira ${ }^{c}$, Rui M.V. Abreu ${ }^{c}$, \\ Maria José Alves ${ }^{c}$, Ricardo C. Calhelha ${ }^{c}$, Adelar Bracht ${ }^{\mathrm{a}, \mathrm{b}}$, Rosane M. Peralta ${ }^{\mathrm{a}, \mathrm{b}, *}$, \\ Isabel C.F.R. Ferreira, ${ }^{\mathrm{c}, *}$ \\ ${ }^{a}$ Department of Biochemistry, State University of Maringá, Paraná, Brazil \\ ${ }^{\mathrm{b}}$ Graduate Program in Food Science, State University of Maringá, Paraná, Brazil \\ ${ }^{\mathrm{c}}$ Centro de Investigação de Montanha (CIMO), Instituto Politécnico de Bragança, Campus de Santa Apolónia, 5300-253 Bragança, Portugal
}

\section{A R T I C L E I N F O}

\section{Keywords:}

Euterpe edulis

Residue

Nutritional value

Antioxidant potential

Antimicrobial activity

Phenolic compounds

\begin{abstract}
A B S T R A C T
The pulp of the fruits of Juçara (Euterpes edulis Martius), a native tree of the Atlantic Rainforest of Brazil, is widely consumed thanks to its flavour and nutritional value. The industrial production of Juçara fruit pulp generates solid residues (peel) which are usually discarded. In this work, a hydroalcoholic extract from Juçara peel flour was evaluated for its phenolic profile as well as for its bioactivities. A total of nineteen phenolic compounds were identified in the Juçara peel flour. Among these, seventeen were non-anthocyanin phenolic compounds, namely two phenolic acids, four flavanonols, six flavones, and five flavonols; whereas the two anthocyanin molecules were cyanidin glycoside derivatives. The Euterpe edulis peel flour presented antioxidant activity and antibacterial potential but was not hepatotoxic. These observations corroborate the idea that this byproduct could fit well into the circular bioeconomy concept, thus promoting the Juçara fruit production chain.
\end{abstract}

\section{Introduction}

Euterpe edulis Martius, popularly known as Juçara, is a native tree of the Atlantic Rainforest found predominantly in the states of the southern and southeastern regions of Brazil (Schulz, Borges, Gonzaga, Costa, \& Fett, 2016). It produces a noble type of palm heart and much appreciated berries (Fig. 1). The palm hearts are largely consumed and of economic relevance in Brazil, presenting superior quality and flavour compared to other species of the genus Euterpe (Borges et al., 2013). In turn, the Juçara fruit is a globose berry that weighs about $1 \mathrm{~g}(90 \%$ of which corresponds to the single seed) that, when ripe, acquires a dark purple shade that closely resembles the fruits of Euterpe oleracea Mart. and Euterpe precatoria Mart. employed in the production of açaí (Schulz et al., 2017). However, the $E$. edulis fruit has a sweeter taste than these last two, being very appreciated by açaí consumers (Felzenszwalb, da Costa Marques, Mazzei, \& Aiub, 2013).

Several authors have demonstrated the exceptional nutritional characteristics of Juçara berries and described their main bioactive constituents, such as flavonoids, especially anthocyanins, and phenolic acids, to which their antioxidant activity is attributed (Inada et al.,
2015; Schulz et al., 2016; Vieira, Marques, Machado, Silva, \& Hubinger, 2017). The extraordinary antioxidant potential of $E$. edulis fruits has been largely confirmed in in vitro (Bicudo, Ribani, \& Beta, 2014; Cardoso et al., 2015; Schulz et al., 2015), ex-vivo (Borges et al., 2013) and in vivo studies (Freitas et al., 2016). Furthermore, a clinical trial verified that the Juçara juice consumption had a very positive action on the antioxidant status and cellular oxidative damage of healthy volunteers (Cardoso et al., 2015). In recent years, the Juçara pulp has been applied as a substrate for probiotic bacteria fermentation (Guergoletto, Mauro, \& Garcia, 2017). The incorporation of this ingredient in yogurt formulation enhanced the nutritional status of the product as well as the probiotic resistance to simulated gastric and enteric conditions (Geraldi, Tulini, Souza, \& De Martinis, 2018).

Juçara berries are almost exclusively commercialized as frozen pulp, which is consumed as such or used as the main ingredient of various cold beverages and ice creams (Schulz et al., 2016). For commercial application, the fruits are macerated and blended with different amounts of water in a depulping apparatus, where the epicarp and mesocarp are separated from the seeds. Consequently, the industrial production of Juçara pulp generates solid residues and effluents, which

\footnotetext{
* Corresponding authors at: Department of Biochemistry, State University of Maringá, Paraná, Brazil (R.M. Peralta).

E-mail addresses: peralta@uem.br (R.M. Peralta), iferreira@ipb.pt (I.C.F.R. Ferreira).
} 


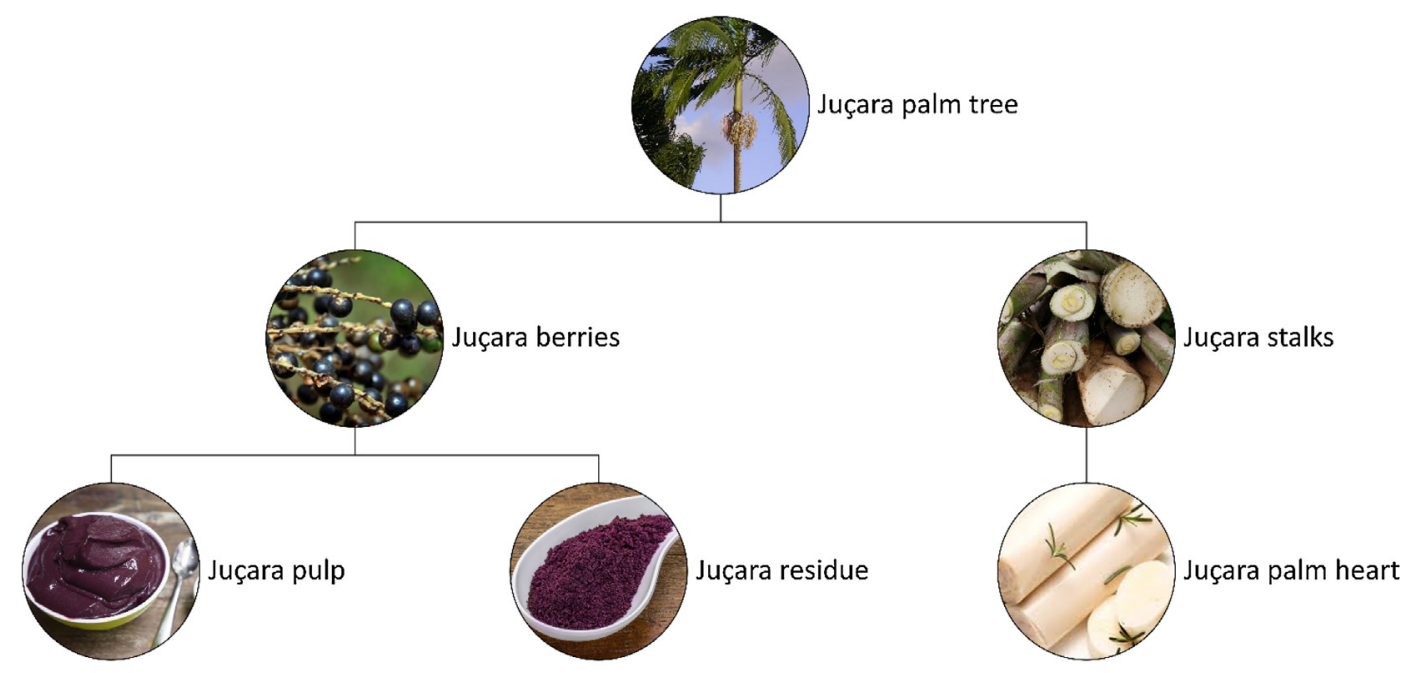

Fig. 1. Juçara palm (Euterpe edulis Martus) major products and by-product.

are often discarded (Ribeiro et al., 2018). Nevertheless, the recovery of antioxidant compounds from Juçara bio-residues have been performed via different methodologies, such as pressurized liquids, supercritical fluids (del Pilar Garcia-Mendoza et al., 2017), thermal assisted extraction (Ribeiro et al., 2018) and nanofiltration membranes (Vieira et al., 2018), along with the identification of its main anthocyanin compounds. Thus, a more detailed characterization of the bioactive components present in these by-products is still lacking.

Employing native plants as part of local food production can help to create a more sustainable agriculture, which runs in parallel with the preservation of biodiversity (Shelef, Weisberg, \& Provenza, 2017). However, any system aiming at preserving biodiversity must be economically viable (Schulz et al., 2016). Thus, supporting the Juçara fruit production chain is fundamental not only to the sustainable handling of E. edulis and the Atlantic Forest biome, but also to ensure an important source of income to the local family agro-industry. In this sense, it is crucial to expand the possibilities of exploitation and valorisation of the bio-waste generated by the processing of Juçara berries.

Based on the above considerations, the aim of the present work was to perform an in-depth study on the bioactive components profile of the Euterpe edulis Martius fruit peel. The nutritional composition of this material was estimated and its hydroethanolic extract was characterized in terms of phenolic compounds, besides antioxidant and antibacterial potentials. Finally, the hepatotoxicity of the extract was assessed. The results are expected to widen the knowledge on the potentialities of this agro-industrial by-product as a source of high added value molecules.

\section{Materials and methods}

\subsection{Euterpe edulis peel flour and nutritional characterization}

E. edulis berries were harvested in the city of Marialva (Paraná, Brazil) and immediately taken to the Laboratory of Biochemistry of Microorganisms of the State University of Maringa, where they were sorted to exclude those unripe or with cracks. Next, the berries were washed in running potable water and sanitized with sodium hypochlorite $(200.0 \mathrm{mg} / \mathrm{L})$ by immersion for $10 \mathrm{~min}$. Then the berries were manually de-pulped and peels were separated from the pulp and seeds. The aim of this procedure was to mimic the agro-industrial residue generation common during Juçara fruits processing into pulp. The obtained peels were submitted to drying $\left(40 \pm 5{ }^{\circ} \mathrm{C}\right)$ for $24 \mathrm{~h}$ in a forced air circulation oven, being later mashed in a blender and sieved (80 mesh) to obtain a homogeneous flour. Finally, the material was stored away from light and oxygen at room temperature $\left(28^{\circ} \mathrm{C}\right)$.
The proximate composition of the E. edulis peel flour (moisture, protein, fat, ash and carbohydrates) was determined using standard procedures (AOAC, 2016). The crude protein content $(\mathrm{N} \times 6.25)$ was estimated by the macro-Kjeldahl method, whereas the crude fat was determined by extracting a known weight of powdered sample with petroleum ether, using a Soxhlet apparatus. The ash content was determined by incineration at $550 \pm 15^{\circ} \mathrm{C}$; total fiber was determined by an enzymatic-gravimetric method and finally the carbohydrate content was calculated by difference. Lastly, the total energy was calculated according to the following equation: Energy $(\mathrm{kcal})=4 \times\left(\mathrm{g}_{\text {proteins }}+\mathrm{g}_{\text {carbohydrates }}\right)+9 \times\left(\mathrm{g}_{\text {lipids }}\right)$.

\subsection{Extract preparation}

The extract was prepared using a ratio flour/solvent of 1:20, with an extractor solution composed of $70 \%$ ethanol in deionized water. The vials were sealed and shaken for $2 \mathrm{~h}$ at $130 \mathrm{rpm}$, at room temperature and protected from light. This procedure was repeated three times. Thereupon the samples were centrifuged at $10^{\circ} \mathrm{C}$ and $10,000 \mathrm{rpm}$ during $15 \mathrm{~min}$, and the obtained supernatants were evaporated at $45^{\circ} \mathrm{C}$ to remove ethanol. In the end, the aqueous phase was lyophilized and stored in a freezer at $-20^{\circ} \mathrm{C}$.

\subsection{Phenolic compounds analyses}

\subsubsection{Non-anthocyanin compounds}

To remove the interfering compounds present in the extract (such as sugars), the extract was purified using a C- 18 SepPak $^{\circledast}$ Vac 3 cartridge (Phenomenex, Torrance, CA, USA). The activation was performed with $5 \mathrm{~mL}$ of ethanol and water, respectively, and then $10 \mathrm{~mL}$ of the sample $(50 \mathrm{mg} / \mathrm{mL}$ ) was loaded into the cartridge. Thereupon the sugars and the more polar compounds were removed by passing $15 \mathrm{~mL}$ water and the phenolic compounds were further eluted with $15 \mathrm{~mL}$ of ethanol. Afterwards, the ethanol was removed under vacuum until dryness and re-dissolved in $1 \mathrm{~mL}$ of $80 \%$ aqueous ethanol, filtered through a $0.22 \mu \mathrm{m}$ disposable LC filter disk into a $1.5 \mathrm{~mL}$ amber vial for HPLC analysis (Rodrigues et al., 2012). This procedure is fundamental to improve the resolution of the peaks and the separation efficiency of the chromatographic run (Vieira et al., 2017).

The analysis was performed using a chromatographic system Dionex Ultimate 3000 UPLC (Thermo Scientific, San Jose, CA, USA). Detections were performed simultaneously with a DAD $(280,330$, and $370 \mathrm{~nm})$ and with a mass spectrometer (Linear Ion Trap LTQ XL, Thermo Finnigan, San Jose, CA, USA) equipped with an ESI source and operating in negative mode, according to the procedure established by the 
authors (Bessada, Barreira, Barros, Ferreira, \& Oliveira, 2016). The compounds were detected comparing the retention times, UV-Vis and mass spectra data with available standards and literature review. Quantification was accomplished using calibration curves of available phenolic standards based on the UV signal. However, in the case of unavailable commercial standards the calibration curve of the most similar standard available was adopted for quantification. The results were expressed as $\mu \mathrm{g}$ per $\mathrm{g}$ of extract.

\subsubsection{Anthocyanin compounds}

This evaluation was performed using the extracted powder at a concentration of $30 \mathrm{mg} / \mathrm{mL}$ re-dissolved in $80 \%$ aqueous ethanol, filtered through a $0.22 \mu \mathrm{m}$ disposable LC filter disk into a $1.5 \mathrm{~mL}$ amber vial for HPLC analysis. The analysis was made via a UPLC-DAD-ESI/ MSn system (Thermo Finnigan, San Jose, CA, USA), following a methodology described by Gonçalves et al. (2017). Detection was carried out employing a DAD $(520 \mathrm{~nm})$ and with a mass spectrometer (Linear Ion Trap LTQ XL Thermo Finnigan) equipped with an ESI source and operating in positive mode. Compounds identification was accomplished by the retention time, UV-VIS and mass spectra data in comparison with available standards and literature review. Quantitative analysis was carried out with calibration curves of available phenolic standards based on the UV signal. In the case of unavailable commercial standards, the calibration curve of the most similar available standard was used for quantification. The results were expressed as $\mathrm{mg} / \mathrm{g}$ of extract.

\subsection{Antioxidant activity evaluation}

To assess the antioxidant activity of the E. edulis peel extract, five methods were employed: two cell-based methods (1) oxidative haemolysis inhibition assay (OxHLIA); (2) the inhibition of the production of thiobarbituric acid reactive substances (TBARS); besides three traditional in vitro assays (3) reduction of the 2,2-diphenyl-1-picrylhydrazyl radical (DPPH, Sigma-Aldrich, St. Louis, MO, USA); (4) reduction of the 2,2-azino-bis (3-ethylbenzothiazoline-6-sulphonate) cation (ABTS, Sigma-Aldrich, St. Louis, MO, USA), and (5) reduction power of the ferric ion (FRAP). For all tests, successive dilutions of the stock solution were prepared and Trolox (6-hydroxy-2,5,7,8-tetramethylchromane-2-carboxylic acid, Sigma-Aldrich, St. Louis, MO, USA) was employed as a positive control.

For the OxHLIA assay, erythrocytes were obtained as described by Evans et al. (2013). Sheep blood samples were collected from healthy animals and centrifuged at $1000 \mathrm{~g}\left(5 \mathrm{~min}, 10^{\circ} \mathrm{C}\right)$. Plasma and buffy coats were discarded and erythrocytes were first washed once with $\mathrm{NaCl}$ $(150 \mathrm{mM})$ and three times with phosphate-buffered saline (PBS, $\mathrm{pH}$ 7.4). The erythrocyte pellet was resuspended in PBS at $2.8 \%(\mathrm{v} / \mathrm{v})$. Using a flat bottom 48 -well microplate, $200 \mu \mathrm{L}$ of erythrocyte suspension was mixed with $400 \mu \mathrm{L}$ of either PBS solution (control), antioxidant samples dissolved in PBS, or water (for complete haemolysis). After preincubation at $37^{\circ} \mathrm{C}$ for 10 min under shaking, AAPH $(200 \mu \mathrm{L}, 160 \mathrm{mM}$ in PBS) was added and the microplate was incubated under the same conditions. The optical density was then measured at $690 \mathrm{~nm}$ every 10 min (Takebayashi, Iwahashi, Ishimi, \& Tai, 2012). The percentage of the erythrocyte population that remained intact $(\mathrm{P})$ was calculated as follows:

$\mathrm{P}(\%)=\left(\mathrm{S}_{\mathrm{t}}-\mathrm{CH}_{0} / \mathrm{S}_{0}-\mathrm{CH}_{0}\right) \times 100$

where $S_{t}$ and $S_{0}$ correspond to the optical density of the sample at $t$ and 0 min, respectively, and $\mathrm{CH}_{0}$ is the optical density of the complete haemolysis at $0 \mathrm{~min}$.

The results were expressed as delayed time of haemolysis $(\Delta t)$, which was calculated as follows:

$\Delta \mathrm{t}(\min )=\mathrm{Ht}_{50}($ sample $)-\mathrm{Ht}_{50}($ control $)$

where $\mathrm{Ht}_{50}$ is the $50 \%$ haemolytic time (min) graphically obtained from the haemolysis curve of each sample concentration.
The $\Delta \mathrm{t}$ values were then correlated to the different sample concentrations (Takebayashi et al., 2012) and, from the obtained correlation, the inhibitory concentration ( $\mathrm{IC}_{50}$ value, $\mathrm{mg} / \mathrm{mL}$ ) able to promote a $\Delta \mathrm{t}$ haemolysis delay of $60 \mathrm{~min}\left(\mathrm{IC}_{50}(60 \mathrm{~min}) \mu \mathrm{g} / \mathrm{mL}\right.$ ) and $120 \mathrm{~min}\left(\mathrm{IC}_{50}\right.$ (120 min), $\mu \mathrm{g} / \mathrm{mL}$ ) were calculated.

Inhibition of the generation of thiobarbituric acid reactive substances (TBARS), besides DPPH and ABTS activities, were estimated as reported by Corrêa et al. (2015). The malondialdehyde-thiobarbituric acid (MDA-TBA) colour intensity was measured at $532 \mathrm{~nm}$. Results were calculated as inhibition ratio (\%) through the equation $\left[\left(\mathrm{A}_{\text {Control }}-\mathrm{A}_{\text {Sample }}\right) / \mathrm{A}_{\text {Control }}\right] \times 100$ and were expressed as $\mathrm{IC}_{50}$ values. FRAP assay was conducted as described by Koehnlein et al. (2016). Standard curves were constructed employing Trolox $\left(R^{2}=0.999\right)$, thus the results were expressed as $\mu \mathrm{mol}$ Trolox equivalents (TE)/mg of lyophilized extract.

\subsection{Hepatotoxicity evaluation}

The Euterpe edulis peel flour extract was dissolved in water at $4 \mathrm{mg}$ / $\mathrm{mL}$ and then submitted to further dilutions. A cell culture (named PLP2) was prepared using a freshly harvested porcine liver acquired from a local slaughterhouse, following a procedure established by the authors (Abreu et al., 2011). The sulforhodamine B assay was performed to evaluate the extract hepatotoxic potential. Ellipticine (Sigma-Aldrich, St. Louis, MO, USA) was employed as positive control and the result was expressed in $\mathrm{GI}_{50}$ values, which is the concentration that inhibited $50 \%$ of the net cell growth.

\subsection{Antibacterial activity evaluation}

The microbial strains were donated clinical isolates obtained from patients hospitalized in various departments at the Hospital Center of Trás-os-Montes and Alto Douro (Vila Real, Portugal); therefore, there was no direct contact with the patients. Five Gram-negative bacteria (Escherichia coli, Klebsiella pneumoniae, Morganella morganii, Proteus mirabilis and Pseudomonas aeruginosa) and three Gram-positive bacteria (Enterococcus faecalis, Listeria monocytogenes and methicillin-resistant Staphylococcus aureus) were used to access this assay. Minimal inhibitory concentration (MIC) for all the bacterial strains was conducted using a colorimetric assay as described by Pires et al. (2018). The MIC value was defined as the lowest concentration that inhibits the visible bacterial growth, which is evidenced by the change in coloration from yellow to pink in case the microorganism is viable. Minimal bactericidal concentration (MBC) was also determined, by measuring the lowest concentration that yielded no growth, therefore $\mathrm{MBC}$ was defined as the lowest concentration required to kill a bacterium. For the Gram-negative bacteria, antibiotics, such as ampicillin and imipenem, were used as positive controls, while ampicillin and vancomycin were used for the Gram-positive bacteria.

\subsection{Statistical analysis}

All results were expressed as mean values and standard deviations (SD), as an outcome of the three repetitions of the samples and concentrations that were used in all assays.

\section{Results and discussion}

\subsection{Nutritional characterization of the E. edulis peel}

Table 1 shows the nutritional composition of the Juçara peel flour used to obtain the extract investigated in this study. The contents of fat (7.41\%), protein $(7.12 \%)$, and ash $(2.39 \%)$ of the peel sample were very similar to the ones found by Inada et al. (2015), which reported values of $6.9 \%, 5.0 \%$ and $2.5 \%$, respectively, when evaluating the Juçara whole fruit. However, for the Juçara pulp, the authors found much 
Table 1

Nutritional characterization of the Euterpe edulis peel flour, expressed on a dry weight basis (mean $\pm \mathrm{SD}$ ).

\begin{tabular}{ll}
\hline & Euterpe edulis peel flour \\
\hline Fat $(\mathrm{g} / 100 \mathrm{~g})$ & $7.4 \pm 0.1$ \\
Protein $(\mathrm{g} / 100 \mathrm{~g})$ & $7.1 \pm 0.3$ \\
Ash $(\mathrm{g} / 100 \mathrm{~g})$ & $2.4 \pm 0.2$ \\
Carbohydrate $(\mathrm{g} / 100 \mathrm{~g})$ & $43.0 \pm 0.5$ \\
Total fiber $(\mathrm{g} / 100 \mathrm{~g})$ & $40.1 \pm 0.2$ \\
Energy $(\mathrm{kcal} / 100 \mathrm{~g})$ & $267 \pm 0.3$ \\
\hline
\end{tabular}

higher contents of fat (46.6\%) and ash (3.4\%), whereas a very close value of carbohydrate content (42.5\%) was obtained. Silva, Rodrigues, Mercadante, and de Rosso (2014) reported a significantly lower carbohydrate content (28.3\%) for a sample of Juçara pulp.

As expected, the E. edulis peel showed a high fiber content $(40.10 \%)$ and considerable energy density ( $2.67 \mathrm{kcal} / \mathrm{g}$ d.w.), especially due to its lipid content. However, the fiber content was not greater than the total fiber content of the whole fruit (71.8\%) found by Inada et al. (2015). Schulz et al. (2015), when evaluating the chemical composition of the Juçara whole fruit during ripening, also found protein contents $(6.6-7 \%)$ in the same range of the value verified in our peel flour. The fat content of the Juçara fruit varied from 7.06 to $22.07 \%$ throughout the 69-days of monitoring.

Different edaphoclimatic conditions and ripening stages, besides the distinct composition presented by each part of the fruit (added to the non-standardized composition of the manufactured pulp), may explain the discrepancies of nutritional composition between the Juçara fruit samples herein discussed.

\subsection{Phenolic compounds of the E. edulis peel extract}

The phenolic compounds composition and corresponding quantification of the hydroethanolic peel extract of $E$. edulis is presented in Table 2. A total of nineteen phenolic compounds were identified, among which seventeen non-anthocyanins phenolic compounds, such as two phenolic acids, four flavanonols, six flavones, and five flavonols and two anthocyanin molecules (cyanidin glycoside derivatives).

Regarding the phenolic acids, caffeic acid (peak 5) was identified taking into account its $\mathrm{MS}^{2}$ fragmentation pattern and UV-Vis spectra in comparison with a commercial standard. Peak $1\left([\mathrm{M}-\mathrm{H}]^{-}\right.$at $m / z$ 501) was identified as a ferulic acid derivative, presented a $\mathrm{MS}^{2}$ fragment at $\mathrm{m} / \mathrm{z} 193\left([\text { ferulic acid }-\mathrm{H}]^{-}\right.$) from the loss of a hexosyldeoxyhexosyl moiety $(-308 \mathrm{u}$ ), being tentatively assigned as ferulic acid hexosyl-deoxyhexoside. Thus, the exact sugar moiety and location was not possible to identify because their retention times did not correspond to any of the available standards. These findings were taken into consideration in the identification of the other phenolic compounds that will be described.

Four flavanonol derivatives were identified, corresponding to three dihydroquercetin (taxifolin) and one dihydrokaempferol (aromadendrin) glycoside derivatives. Peaks $3\left([\mathrm{M}-\mathrm{H}]^{-}\right.$at $\left.m / z 611\right)$, and 4 and 6 $\left([\mathrm{M}-\mathrm{H}]^{-}\right.$at $m / z$ 465) released an $\mathrm{MS}^{2}$ fragment at $\mathrm{m} / \mathrm{z} 303$ $\left([\text { taxifolin }-\mathrm{H}]^{-}\right)$from the loss of a rutinosyl $(-308 \mathrm{u})$ and hexosyl moiety, respectively, being tentatively identified as taxifolin-O-rutinoside and taxifolin- $O$-hexoside isomers 1 and 2 . The $\mathrm{MS}^{2}$ fragmentation pattern of peak $2\left([\mathrm{M}-\mathrm{H}]^{-}\right.$at $\left.m / z 581\right)$ indicated that it corresponds to a aromadendrin derivative bearing pentosyl and hexosyl residues. The observation of only one $\mathrm{MS}^{2}$ fragment at $\mathrm{m} / \mathrm{z} 287$ ( -132 to $162 \mathrm{u}$ ), suggested that the two sugars were linked together. Thus, they were tentatively identified as aromadendrin- $O$-pentosyl-hexoside.

A total of six flavones (peaks 7-9, 12, 15, and 16) were identified in this hydroethanolic peel extract, in which peaks 7, 8, 9 and 12 presented similar UV spectra suggesting that they may derive from apigenin, as they are also showing ion fragments characteristic of $\mathrm{C}$ - glycosylated flavones (Ferreres et al., 2012; Pereira et al., 2014). Peaks 7,8 , and 9 showed the same pseudomolecular ion $[\mathrm{M}-\mathrm{H}]^{-}$at $m / z 563$ pointing to an apigenin bearing a pentosyl $(-90 \mathrm{u})$ and a hexosyl $(-120 \mathrm{mu})$ sugar substituents, but yielded different $\mathrm{MS}^{2}$ fragmentation intensities. Thus, taking into account the findings reported by Ferreres et al. (2012) and Pereira et al. (2014), these peaks were tentatively assigned as apigenin-6- $C$-pentoside- 8 - $C$-hexoside isomer 1 and 2 (peaks 7 and 8 ) and apigenin-6-C-hexiside- 8 - $C$-pentoside (peak 9). Compound $12\left([\mathrm{M}-\mathrm{H}]^{-}\right.$at $\left.m / z 499\right)$ presented a fragmentation pattern similar to apigenin- $C$-glucoside, thus the losses of $-44 \mathrm{u}$ and $-24 \mathrm{u}$ from the fragments $\mathrm{m} / \mathrm{z} 455$ and 431 do not have a possible identification, therefore, this compound was identified as a apigenin- $C$-glucoside derivative. The remaining flavone derivatives (peaks 15 and 16), derived from hispidulin ( $\lambda_{\max }$ around $335 \mathrm{~nm}$ and an $\mathrm{MS}^{2}$ fragment at $m / z 299$ ). Peak $15\left([\mathrm{M}-\mathrm{H}]^{-}\right.$at $\left.m / z 607\right)$ and peak $16\left([\mathrm{M}-\mathrm{H}]^{-}\right.$at $\left.m / z 461\right)$ presented a $\mathrm{MS}^{2}$ fragmention pattern corresponding to the loss of a rutinosyl ( $-308 \mathrm{mu}$ ) and hexosyl $(-162 \mathrm{u})$ residue, respectively. Thus, as mentioned above, the nature and position of the sugar moieties could not be identified; therefore, they were tentatively assigned to hispidulin-O-rutinoside and hispidulin- $O$-hexoside, respectively.

Five flavonols were identified, being quercetin-3-O-rutinoside (peak 11), kaempferol-3-O-rutinoside (peak 13), and isorhamnetin-3-O-rutinoside (peak 14) positively identified by comparison with commercial standards. Compound $10\left([\mathrm{M}-\mathrm{H}]^{-}\right.$at $\left.m / z 593\right)$ presented the same pseudomolecular ion as peak 13, identified as kaempferol-3-O-rutinoside, thus presenting a different retention time. As no information about the identity of the sugar moieties and location onto the aglycone could be obtained, the compound was tentatively identified as kaempferol-Ohexosyl-deoxyhexoside. The mass characteristics of peak $17\left([\mathrm{M}-\mathrm{H}]^{-}\right.$ at $m / z 491$ ) presented two $\mathrm{MS}^{2}$ fragments at $m / z 329(-162 \mathrm{u}$ ), bearing a hexosyl residue, and at $m / z 314$ ([isorhamnetin $-2 \mathrm{H}]^{-}$) from the loss of a methyl $(-15 \mathrm{u})$ moiety, thus being tentatively assigned to a methylisorhamnetin- $O$-hexoside.

Among the non-anthocyanins compounds, apigenin-6- $C$-pentoside8- $C$-hexoside isomers were the dominant components, representing $23 \%$ of the total phenolic non-anthocyanin compounds, followed by a apigenin- $C$-glucoside derivative (11.84\%), kaempferol-O-hexosyldeoxyhexoside (7.54\%), and taxifolin-O-hexoside isomer 2 (7.18\%).

Different non-anthocyanin phenolic compounds have been reported in literature, 15 phenolic acids being identified (above all gallic, protocatechuic, $p$-coumaric and ferulic acids); 13 flavonoids (primarily quercetin derivatives, such as rutin); and one stilbene (resveratrol) (Bicudo et al., 2014; Borges et al., 2013; Cardoso et al., 2015; Inada et al., 2015; Schulz et al., 2015, 2016). Moreover, Vieira et al. (2017) identified similar compounds in a hydroethanolic extract of Juçara pulp using an UPLC/ESI-MS, including five flavonols (quercetin, rutin, myricetin, kaempferol, and kaempferol-3-rutinoside), two flavones (luteolin and apigenin), one flavanol (catechin), and two phenolic acids (ellagic acid, and 4,5-dicaffeoylquinic acid). Schulz et al. (2017), in their study on the bioaccessibility of Juçara fruits bioactive compounds subjected to in vitro gastrointestinal digestion, also detected aromadendrin (21.6-164.3 $\mu \mathrm{g} / \mathrm{g}$ ) and ferulic (25.9-181.2 $\mu \mathrm{g} / \mathrm{g}$ ) acids through a LC-ESI-MS/MS analysis. Besides, protocatechuic $(85.7-229.4 \mu \mathrm{g} / \mathrm{g}$ ) and vanillic (85.1-165.1 $\mu \mathrm{g} / \mathrm{g}$ ) acids were also identified. Both aromadendrin and ferulic acid contents (59.13 and $47.2 \mu \mathrm{g} / \mathrm{g}$, respectively) found in our peel extract were within the ranges reported by these authors. Although there were similarities regarding the mentioned compounds or main identified aglycones, the non-anthocyanin phenolic profile of our sample was quite different from the one reported by these authors. This fact can perhaps be explained not only by the different edafoclimatic conditions but also by the defattering and extraction procedures performed by the authors.

The remaining compounds corresponded to anthocyanins. Peak 19 was positively identified as cyanidin-3-O-glucoside in comparison with the chromatographic characteristics of the commercial standard. Peak $18\left([\mathrm{M}+\mathrm{H}]^{+}\right.$at $\left.m / z 595\right)$ presented a unique $\mathrm{MS}^{2}$ fragment at $m / z 287$ 
Table 2

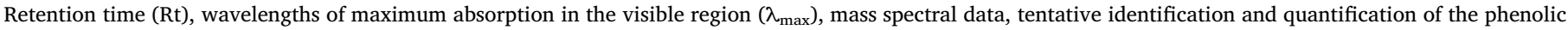
compounds present in the hydroethanolic extract from Euterpe edulis peels (mean $\pm \mathrm{SD}$ ).

\begin{tabular}{|c|c|c|c|c|c|c|}
\hline Peak & Rt (min) & $\lambda \max (\mathrm{nm})$ & $\begin{array}{l}{[\mathrm{M}-\mathrm{H}]^{-}(m /} \\
z)\end{array}$ & $\operatorname{MS}^{2}(m / z)$ & Tentative identification & $\begin{array}{l}\text { Quantification ( } \mu \mathrm{g} / \\
\mathrm{g})\end{array}$ \\
\hline 1 & 5.1 & 320 & 501 & 193(100) & Ferulic acid hexosyl-deoxyhexoside $\mathrm{A}^{(\mathrm{A})}$ & $47.2 \pm 0.9$ \\
\hline 2 & 5.54 & $274, \operatorname{sh} 330$ & 581 & $287(100)$ & Aromadendrin-O-pentosyl-hexoside ${ }^{(\mathrm{B})}$ & $59.13 \pm 0.01$ \\
\hline 3 & 7.04 & $281, \operatorname{sh} 338$ & 611 & $303(100)$ & Taxifolin-O-rutinoside $\mathrm{e}^{(\mathrm{B})}$ & $25.2 \pm 0.5$ \\
\hline 4 & 7.58 & $281, \operatorname{sh} 340$ & 465 & $303(100)$ & Taxifolin- $O$-hexoside isomer $1^{(\mathrm{B})}$ & $26.5 \pm 0.3$ \\
\hline 5 & 9.33 & 323 & 179 & $135(100)$ & Caffeic $\operatorname{acid}^{(\mathrm{C})}$ & $53 \pm 2$ \\
\hline 6 & 11.4 & $288, \operatorname{sh} 340$ & 465 & $303(100)$ & Taxifolin-O-hexoside isomer $2^{(\mathrm{B})}$ & $122 \pm 6$ \\
\hline 7 & 12.88 & 330 & 563 & $545(22), 503(62), 473(100), 443(65), 383(30), 353(28), 311(5)$ & $\begin{array}{l}\text { Apigenin-6- } C \text {-pentoside-8- } C \text {-hexoside } \\
\text { isomer } 1^{(\mathrm{D})}\end{array}$ & $239 \pm 1$ \\
\hline 8 & 13.7 & 330 & 563 & $545(29), 503(71), 473(100), 443(81), 383(29), 353(29), 311(5)$ & $\begin{array}{l}\text { Apigenin-6- } C \text {-pentoside-8- } C \text {-hexoside } \\
\text { isomer } 2^{(\mathrm{D})}\end{array}$ & $145 \pm 2$ \\
\hline 9 & 13.97 & 330 & 563 & 473(66),443(100),383(12),353(27),311(5) & Apigenin-6-C-hexiside-8-C-pentoside ${ }^{(\mathrm{D})}$ & $175 \pm 2$ \\
\hline 10 & 16.42 & 346 & 593 & $285(100)$ & Kaempferol-O-hexosyl-deoxyhexoside ${ }^{(\mathrm{E})}$ & $128 \pm 4$ \\
\hline 11 & 17.77 & 350 & 609 & $301(100)$ & Quercetin-3-O-rutinoside ${ }^{(\mathrm{E})}$ & $91 \pm 2$ \\
\hline 12 & 19.01 & 335 & 499 & $455(100), 431(18), 413(13), 393(6), 353(7), 311(5)$ & Apigenin- $C$-glucoside derivative ${ }^{(D)}$ & $201 \pm 3$ \\
\hline 13 & 21.09 & 342 & 593 & $285(100)$ & Kaempferol-3-O-rutinoside ${ }^{(\mathrm{E})}$ & $64.5 \pm 0.7$ \\
\hline 14 & 22.08 & 350 & 623 & $315(100)$ & Isorhamnetin-3-O-rutinoside $\mathrm{e}^{\mathrm{E})}$ & $71.0 \pm 0.3$ \\
\hline 15 & 23.06 & 330 & 607 & $299(100)$ & Hispidulin-O-rutinoside $\mathrm{e}^{(\mathrm{E})}$ & $66.7 \pm 3.0$ \\
\hline 16 & 24.82 & 335 & 461 & $299(100)$ & Hispidulin-O-hexoside ${ }^{(\mathrm{E})}$ & $91 \pm 2$ \\
\hline \multirow[t]{2}{*}{17} & 25.40 & 333 & 491 & $329(100), 314(15)$ & MethylIsorhamnetin-O-hexoside ${ }^{(\mathrm{E})}$ & $91.8 \pm 0.7$ \\
\hline & & & & & $\begin{array}{l}\text { TPA } \\
\text { TFNA } \\
\text { TPCNA }\end{array}$ & $\begin{array}{l}100 \pm 3 \\
1598 \pm 5 \\
1698 \pm 2\end{array}$ \\
\hline Peak & Rt (min) & $\lambda \max (\mathrm{nm})$ & $\begin{array}{l}{[\mathrm{M}+\mathrm{H}]^{+}(m /} \\
z)\end{array}$ & $\operatorname{MS}^{2}(m / z)$ & Tentative identification & $\begin{array}{l}\text { Quantification (mg/ } \\
\text { g) }\end{array}$ \\
\hline 18 & 23.12 & 524 & 595 & $449(54), 287(100)$ & Cyanidin-3-O-rutinoside ${ }^{(\mathrm{F})}$ & $5.32 \pm 0.01$ \\
\hline \multirow[t]{2}{*}{19} & 24.75 & 522 & 449 & $287(100)$ & Cyanidin-3-O-glucoside ${ }^{(\mathrm{F})}$ & $6.23 \pm 0.03$ \\
\hline & & & & & TA & $11.54 \pm 0.02$ \\
\hline
\end{tabular}

TPA - total phenolic acids; TFNA - total flavonoids non-anthocyanins; TPCNA - total phenolic compounds non-anthocyanins; TA - total anthocyanins. Standard calibration curves: A - ferulic acid $\left(y=633126 x-185462, R^{2}=0.999\right) ; \mathrm{B}-$ taxifolin $\left(y=203766 x-208383, R^{2}=1\right)$; C - caffeic acid $(y=388345 x+406369$, $\left.R^{2}=0.994\right) ; \mathrm{D}$ - apigenin-6-glucoside $\left(y=107025 x+61531, R^{2}=0.999\right) ; \mathrm{E}-$ quercetin-3-O-glucoside $\left(\mathrm{y}=34843 \mathrm{x}-160173, R^{2}=0.999\right) ; \mathrm{F}-$ cyanidin-3-Oglucoside (y = 134578x - 3,000,000; $\left.R^{2}=0.999\right)$.

(cyanidin; $[\mathrm{M}+\mathrm{H}-308]^{-}$loss of a rutinosyl moiety), being assigned to cyanidin-3-O-rutinoside. The major components of the peel extract were the anthocyanins cyanidin-3-O-rutinoside (C3R) and cyanidin-3$O$-glucoside (C3G) (5.32 and $6.23 \mathrm{mg} / \mathrm{g}$, respectively), which together accounted for more than $87 \%$ of the extract's total phenolic content (TPC), thus corroborating literature data (Bicudo et al., 2014; Borges et al., 2013; Cardoso et al., 2015; Inada et al., 2015; Schulz et al., 2015, 2016).

In the past few years, extensive work has been done regarding the chromatographic phenolic characterization of Juçara's whole fruit and processed pulp. In addition to the C3R and C3G main anthocyanins, minor anthocyanin compounds such as cyanidin-3-sambubioside, pelargonidin-3-glucoside, cyaniding-3-rhamnoside, pelargonidin-3-rutinoside, cyanidin-3,5-hexoside-pentoside, peonidin-3-rutinoside, delfinidin-3-glucoside, cyanidin-3,5-diglucoside, and malvidin-3-glucoside, have also been identified in Juçara berry samples via HPLC-MS/MS (Bicudo et al., 2014; Cardoso et al., 2015; da Silva et al., 2014; Guergoletto, Costabile, Flores, Garcia, \& Gibson, 2016). Using an UPLC/ESI-MS, Vieira et al. (2017) identified a similar set of anthocyanin compounds when analysing a hydroethanolic extract of Juçara pulp, including three anthocyanins (C3R, C3G, and pelargonidin-3-Oglucoside). C3R corresponded to more than $70 \%$ of the Juçara pulp's TPC (about $23 \mathrm{mg} / \mathrm{g}$ of extract in dry weight), more than 4-fold the amount found in our peel extract. However, Bicudo et al., 2014 found lower C3R and C3G contents ( 0.87 and $1.09 \mathrm{mg} / \mathrm{g}$, respectively) when evaluating a methanolic extract of defatted Juçara pulp.

The TPC found (non-anthocyanin and anthocyanin compounds) for our hydroethanolic peel extract was significant $(13.24 \mathrm{mg} / \mathrm{g})$ and is also within the range found by other authors for Juçara fruit residues. Ribeiro et al. (2018), for instance, obtained TPC values varying from
9.59 to $16.23 \mathrm{mg} \mathrm{GAE} / \mathrm{g}$ when investigating the thermal assisted extraction of Juçara residue with different solvents and concentrations (ethanol, methanol and water) at $60^{\circ} \mathrm{C}$, whereas del Pilar GarciaMendoza et al. (2017) reported $51.4 \mathrm{mg} \mathrm{GAE} / \mathrm{g}$ for the Juçara residue extracted with an acidified mixture (pH 2.0) of ethanol + water $50 \%$ (v/v) at $80^{\circ} \mathrm{C}$ (best conditions for pressurized liquid extraction). However, it is well established that other types of compounds (such as reducing sugars) found in great amounts in plant extracts, can also reduce the Folin Ciocalteu reagent, hence generating overestimated values of total phenolic compounds via this method (Sánchez-Rangel, Benavides, Heredia, Cisneros-Zevallos, \& Jacobo-Velázquez, 2013).

\subsection{Evaluation of bioactive properties}

The antioxidant activity, hepatotoxicity, as also the antibacterial properties of the $E$. edulis hydroethanolic peel extract were assessed and the results are shown in Tables $3-5$.

The antioxidant properties are the most frequently reported for $E$. edulis fruits by virtue of the abundance in phenolic compounds, which

\section{Table 3}

Cell-based antioxidant activity of the Euterpe edulis hydroethanolic peel extract and Trolox (positive control) measured via OxHLIA and TBARS formation inhibition assays (mean $\pm \mathrm{SD}$ ).

\begin{tabular}{llll}
\hline & \multicolumn{2}{l}{ OxHLIA } & TBARS \\
\cline { 2 - 3 } & $\mathrm{IC}_{50(60 \mathrm{~min})}, \mu \mathrm{g} / \mathrm{mL}$ & $\mathrm{IC}_{50(120 \mathrm{~min}), \mu \mathrm{g} / \mathrm{mL}}$ & $\mathrm{IC}_{50}, \mu \mathrm{g} / \mathrm{mL}$ \\
\hline Trolox & $5.5 \pm 0.5$ & $20.4 \pm 0.3$ & $9 \pm 1$ \\
E. edulis peel extract & $42 \pm 1$ & $107 \pm 3$ & $204 \pm 14$ \\
\hline
\end{tabular}


Table 4

Antioxidant activity of the Euterpe edulis peel extract evaluated by its capacity in the deactivation of 2,2-diphenyl-1-picrylhydrazyl (DPPH) and 2,2'azino-bis(3-ethylbenzothiazoline-6-sulphonic acid (ABTS) radicals, ferric reducing antioxidant potential (FRAP) and hepatotoxicity (mean \pm SD).

Antioxidant assay (TEAC $\mu \mathrm{mol} \mathrm{TE} / \mathrm{mg}$ )

\begin{tabular}{ll}
\hline DPPH & $13.1 \pm 0.2$ \\
ABTS & $23.2 \pm 0.3$ \\
FRAP & $320 \pm 5$ \\
${\text { Hepatotoxicity }\left(\mathrm{GI}_{50} \mu \mathrm{g} / \mathrm{mL} \text { values }\right)^{\mathrm{A}}}$ \\
\hline PLP2 & $>400$ \\
\hline
\end{tabular}

TEAC - Trolox equivalent antioxidant capacity. $\mathrm{GI}_{50}$ values correspond to the sample concentration achieving $50 \%$ of growth inhibition in liver primary culture PLP2. A - Ellipticine $\mathrm{GI}_{50}$ value: $2.29 \mu \mathrm{g} / \mathrm{mL}$ (PLP2).

are of considerable interest due to their antioxidant and free radicalscavenging abilities (Schulz et al., 2016). Considerable research has been carried out with regard to the in vitro antioxidant potential of $E$. edulis fruit pulp. Its organic extracts have shown significant antioxidant capacities in the deactivation of 2,2-diphenyl-1-picrylhydrazyl (DPPH) (Bicudo et al., 2014; Cardoso et al., 2015; Schulz et al., 2015) and 2,2'azino-bis(3-ethylbenzothiazoline-6-sulphonic acid (ABTS) radicals (Inada et al., 2015), in addition to high ferric reducing antioxidant potential (FRAP) (Cardoso et al., 2015; Inada et al., 2015; Schulz et al., 2015). However, except for the studies of Ribeiro et al. (2018) and del Pilar Garcia-Mendoza et al. (2017), information on the antioxidant capacity of Juçara's fruit residues remain scarce.

The results of the cell-based antioxidant activity assays for the $E$. edulis peel extract are displayed in Table 3. OxHLIA assay results are expressed as the concentration of extract required to inhibit (delay) $50 \%$ of haemolysis for $60 \mathrm{~min}\left(\mathrm{IC}_{50}(60 \mathrm{~min})\right.$ ) and $120 \mathrm{~min}\left(\mathrm{IC}_{50}(60 \mathrm{~min})\right.$ ). This haemolysis delay time standardization guarantees an easier comparative analysis of the extract antioxidant capability. Fig. 1A (Supplementary material) shows the time course of haemolysis at different and increasing extract concentrations.

The $\mathrm{IC}_{50}(120 \mathrm{~min})$ value obtained was almost 4-fold the $\mathrm{IC}_{50}(60 \mathrm{~min})$ value. For comparison purposes, Trolox, a synthetic antioxidant frequently employed as positive control, was also assessed. As expected, it displayed a superior erythrocyte-protection capacity when compared to the extract, with an $\mathrm{IC}_{50}(60 \mathrm{~min})$ value of $5.5 \pm 0.5 \mu \mathrm{g} / \mathrm{mL}$. The peel extract was also evaluated by the TBARS method and the $\mathrm{IC}_{50}$ value

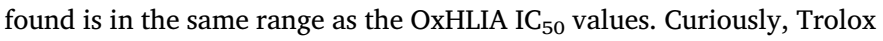
was 22 -fold more potent than the E. edulis extract for the TBARS assay $(9.2 \mu \mathrm{g} / \mathrm{mL}$ compared to $203.59 \mu \mathrm{g} / \mathrm{mL})$, whereas it was only 8 -fold more potent in the OxHLIA method $(5.5 \mu \mathrm{g} / \mathrm{mL}$ compared to $42 \mu \mathrm{g} / \mathrm{mL})$.

Depending on their $\mathrm{IC}_{50}$ values, plant extracts can be classified as highly active $\left(\mathrm{IC}_{50}<50 \mu \mathrm{g} / \mathrm{mL}\right)$; moderately active
$\left(50<\mathrm{IC}_{50}<100 \mu \mathrm{g} / \mathrm{mL}\right)$; weakly active $\left(100<\mathrm{IC}_{50}<200 \mu \mathrm{g} / \mathrm{mL}\right)$ or inactive ( $\mathrm{IC}_{50}>200 \mu \mathrm{g} / \mathrm{mL}$ ) (Rai et al., 2017). Taking these notions into account, in the OxHLIA assay the peel extract displayed high antioxidant activity, however in the TBARS assay it can be classified as inactive.

Table 4 shows the antioxidant capacity of the Juçara fruit peel estimated by the DPPH, ABTS and FRAP assays, all expressed in Trolox equivalent antioxidant capacity (TEAC) values.

Bicudo et al. (2014) reported a much lower DPPH radical scavenging activity $(745 \mu \mathrm{mol} \mathrm{TE} / \mathrm{g})$ for a methanolic extract of the defatted Juçara pulp than the one verified for our peel extract $(13,107 \mu \mathrm{mol}$ TE/ g). However, our sample presented significantly less expressive ABTS radical scavenging activity $(23 \mu \mathrm{mol} \mathrm{TE} / \mathrm{mg})$ than the methanolic pulp extract assessed by Inada et al. (2015) $(677 \mu \mathrm{mol} \mathrm{TE} / \mathrm{mg})$. Borges et al. (2013) and Cardoso et al. (2015) reported inferior antioxidant capacities assessed via FRAP assay for Juçara's whole fruit and pulp extracts (1158 and $2155 \mu \mathrm{mol} \mathrm{TE} / 100 \mathrm{~g}$, respectively) in comparison with our result for this method $(3199 \mu \mathrm{mol} \mathrm{TE} / 100 \mathrm{~g})$.

With respect to the antioxidant capacity of $E$. edulis fruit residues, del Pilar Garcia-Mendoza et al. (2017) verified a considerably lower antioxidant capacity $(0.046 \mu \mathrm{mol} \mathrm{TE} / \mathrm{g})$ using the DPPH method for a Juçara residue sample extracted with an acidified mixture of ethanol/ water $(50 \% \mathrm{v} / \mathrm{v})$ under pressure. Although Ribeiro et al. (2018) had reported a superior antioxidant activity value of $38.96 \mu \mathrm{mol} \mathrm{TE} / \mathrm{g}$ for the ABTS assay, they found a less expressive DPPH activity $(32.85 \mu \mathrm{mol}$ $\mathrm{TE} / \mathrm{g}$ ) when evaluating an aqueous extract of $E$. edulis residue obtained via thermal assisted extraction. It is worth to mention that both extraction technologies used by these groups enable the recovery of greater amounts of phenolic compounds.

Anthocyanins are amidst the main compounds related to the great free radical-scavenging capacities of Juçara fruit, whereas a significant positive correlation with antioxidant capacity was observed (Bicudo et al., 2014; Schulz et al., 2015, 2016). Scientific studies using cell culture, animal models, and human clinical trials, have largely demonstrated that anthocyanins own pronounced antioxidative and antimicrobial potentials, ameliorate visual and neurological health, and protect against diverse non-communicable illnesses. Such health benefits are attributed mainly to their strong antioxidant properties. Among the distinct mechanisms and pathways involved in anthocyanins' protective outcomes, there are the free-radical scavenging, cyclooxygenase and mitogen-activated protein kinase pathways as well as the inflammatory cytokines signalling (Khoo, Azlan, Tang, \& Lim, 2017). Substantial evidence has shown that C3G, the major anthocyanin found in most plants, as also the main compound present in E. edulis residue, holds antioxidant, anti-inflammatory, cardio-protective, anti-diabetic, and anti-cancer properties both in vitro and in vivo (Petroni et al., 2017; Rupasinghe, Arumuggam, Amararathna, \& De Silva, 2018).

Furthermore, the major non-anthocyanin components found in the peel extract, apigenin $C$-glycoside derivatives, are promising bioactive

Table 5

Antibacterial potential of the Euterpe edulis peel extract.

\begin{tabular}{|c|c|c|c|c|c|c|c|c|}
\hline & \multicolumn{2}{|c|}{ E. edulis } & \multicolumn{2}{|c|}{ Ampicillin } & \multicolumn{2}{|l|}{ Imipenem } & \multicolumn{2}{|c|}{ Vancomycin } \\
\hline & MIC & MBC & MIC & MBC & MIC & MBC & MIC & MBC \\
\hline \multicolumn{9}{|l|}{ Gram-negative bacteria } \\
\hline Escherichia coli & 5 & $>20$ & $<0.15$ & $<0.15$ & $<0.0078$ & $<0.0078$ & n.t. & n.t. \\
\hline Klebsiella pneumoniae & 20 & $>20$ & 10 & 20 & $<0.0078$ & $<0.0078$ & n.t. & n.t. \\
\hline Morganella morganii & 10 & $>20$ & 20 & $>20$ & $<0.0078$ & $<0.0078$ & n.t. & n.t. \\
\hline Proteus mirabilis & $>20$ & $>20$ & $<0.15$ & $<0.15$ & $<0.0078$ & $<0.0078$ & n.t. & n.t. \\
\hline Pseudomonas aeruginosa & 20 & $>20$ & $>20$ & $>20$ & 0.5 & 1 & n.t. & n.t. \\
\hline \multicolumn{9}{|l|}{ Gram-positive bacteria } \\
\hline Enterococcus faecalis & 10 & $>20$ & $<0.15$ & $<0.15$ & n.t. & n.t. & $<0.0078$ & $<0.0078$ \\
\hline Listeria monocytogenes & 10 & $>20$ & $<0.15$ & $<0.15$ & n.t. & n.t. & n.t. & n.t. \\
\hline MRSA & 5 & $>20$ & $<0.15$ & $<0.15$ & n.t. & n.t. & 0.25 & 0.5 \\
\hline
\end{tabular}

MIC - minimum inhibitory concentration; MBC - minimum bactericidal concentrations; n.t. - not tested; MRSA - Methicillin-resistant Staphylococcus aureus. 
flavonoids. Apigenin, a flavone derivative commonly found in a variety of edible and medicinal plants, has displayed significant anti-inflammatory, anti-carcinogenic and antioxidant potentials in several in vitro and in vivo experimental models (Madunić, Madunić, Gajski, Popić, \& Garaj-Vrhovac, 2018; Wang, Zhou, Zhao, Ye, \& Xie, 2018). In the past few years, this compound has aroused huge interest as a potential chemotherapeutic agent owing to its low intrinsic toxicity and notable results on normal versus cancerous cells, compared with other structurally similar flavonoids (Madunić et al., 2018). Regarding its antioxidant effects, it was verified that apigenin down regulates adhesion molecules (Liu et al., 2011), while suppressing oxidative stress through its direct free radical scavenging action and upregulating intracellular antioxidant defences. Although displaying high antioxidant activity in vitro, assessed via different methods, the low intestinal absorption of apigenin verified in a Caco-2 cell monolayers model, indicates that encapsulation may be mandatory to enhance its bioavailability for applications as oral antioxidant drug/supplement (Pérez-Sánchez et al., 2017).

The evaluated $E$. edulis peel extract showed no toxicity against the liver primary culture PLP2, since the $\mathrm{GI}_{50}$ value was higher than the highest concentration assessed $(400 \mu \mathrm{g} / \mathrm{mL})$ (Table 4$)$.

Felzenszwalb et al. (2013) performed a toxicological evaluation of E. edulis fruits using a broad complex interdisciplinary approach, which included mass spectroscopy analyses, blood biochemistry, genotoxicity, bacterial reverse mutation and cytotoxicity tests. Besides demonstrating that the Juçara fruit has no adverse effects on human health, the authors concluded that its pulp contains compounds with the capacity to generate mutagenicity and clastogenic/aneugenic results. Later, Freitas et al. (2016) investigated the action of three E. edulis products, namely (1) lyophilized pulp; (2) defatted lyophilized pulp, and (3) oil, on nonalcoholic fatty liver disease induced by a high-fat diet in rats. The dietary consumption of the Juçara fruit lyophilized pulps, but not the oil, mitigated the liver disease, minimizing inflammatory infiltrates, steatosis, and lipid peroxidation in the liver tissue. Although none of the analysed products was hepatotoxic, only the defatted pulp promoted sufficient benefits to treat the liver disease in vivo, likely by its low lipid content and high amount of antioxidant phenolic compounds.

The use of novel plant polyphenols in food and pharmaceutical industries mandatorily demands proof of its toxicological properties (Schulz et al., 2016). Therefore, the absence of hepatotoxicity verified herein not only corroborates the above-cited previous reports but also endorses the safe application of Juçara peel's extracts or biomolecules as possible food additives.

Concerning the antimicrobial activity, the values regarding the minimum inhibitory concentration (MIC) of the E. edulis peel extract against five Gram-negative and three Gram-positive bacteria are shown in Table 5. The inhibitory activity displayed by the extract against both Gram-positive (Enterococcus faecalis, Listeria monocytogenes, Methicillinresistant Staphylococcus aureus - MRSA) and Gram-negative (Escherichia coli, Klebsiella pneumonia, Morganella morganii and Pseudomonas aeruginosa) bacteria indicates the presence of an extensive spectrum of phytochemical constituents with antibiotic potential. The E. edulis peel extract was more effective than the antibiotic ampicillin against $M$. morganii and $P$. aeruginosa.

Overall, the hydroethanolic peel extract was more active against Gram-positive bacteria than against Gram-negative bacteria. The same trend was observed by Favaro et al. (2018) when exploring the antibacterial activity of a crude anthocyanin extract of Juçara fruit against $S$. aureus, P. aeruginosa and E. coli via the agar-diffusion method. Although the $E$. edulis anthocyanin extract had displayed a slightly significant effect against $S$. aureus, it had no activity against $E$. coli and $P$. aeruginosa.

Interestingly, anthocyanin-rich extracts (e.g. blueberry, raspberry, blackcurrant, and strawberry extracts) tend to inhibit Gram-negative bacteria; however, they are less efficient against Gram-positive bacteria (Khoo et al., 2017). Thus, the herein observed antimicrobial effects are likely resulting from the multiple mechanisms and synergistic outcomes of diverse bioactive phytochemical constitutes of the Juçara peel extract. Banerjee, Banerjee, Das, and Mandal (2015) studied the antibacterial action of free and a liposomal formulation of apigenin, with promising results. Liposomal apigenin significantly decreased MIC values against both Gram-positive (Bacillus subtilis and $S$. aureus) and Gram-negative ( $P$. aeruginosa and $E$. coli) bacteria in comparison to the free compound. Apigenin is one of the plant-derived phenolic compounds that have been prospected as natural food preservatives in the past years.

The Juçara peel extract presented MIC values above 1.6 for all the bacteria tested, results that some authors classify as a weak inhibitory profile (Corrêa et al., 2018). However, it is essential to highlight that the bacteria strains used in our assays are clinical isolated multi-resistant strains, that present antibiotic resistance profiles quite superior to those of ATCC bacterial strains (Dias et al., 2016). With this in mind, the herein reported data can be interpreted as an indicative of significant antibacterial potential.

\section{Conclusion}

To our best knowledge, this is the first study on the nutritional composition, antibacterial potential and hepatotoxicity of the peels of Juçara fruit. Furthermore, we presented a broad characterization of the peel extract phenolic composition, along with the inedited TPC chromatographic quantification, which revealed its outstanding anthocyanin content. Anthocyanins of berries are relevant compounds not only as potential agents that protect against some illnesses, but also as promising natural food colorants. The herein reported results suggest the viability of exploiting the bio-residue generated by the industrial processing of Juçara berries for the obtainment of bioactive compounds and/or extracts, taking into account the verified in vitro antioxidant and antibacterial potentials, besides lack of hepatotoxicity. Therefore, Juçara fruit residues could be used to produce high added-value food additives, both colorants and preservatives, following the circular bioeconomy concept and stimulating the Juçara fruit production chain.

\section{Conflict of interest}

The authors declare no conflict of interests.

\section{Acknowledgments}

J.A.A. Garcia thanks Coordenação de Aperfeiçoamento de Pessoal de Nível Superior (CAPES) for the financial support provided for her masters studies in the State University of Maringá. R.C.G. Corrêa thanks Conselho Nacional de Desenvolvimento Científico e Tecnologia (CNPq) for financing her postdoctoral research at State University of Maringá (Process number 167378/2017-1). R.M. Peralta (Project number 307944/2015-8) and A. Bracht (Project number 304090/2016-6) are CNPq research grant recipients. The authors are grateful to the Foundation for Science and Technology (FCT, Portugal) and FEDER under Programme PT2020 for financial support to CIMO (UID/AGR/ 00690/2013), L. Barros and R. C. Calhelha contracts; to FEDER-Interreg España-Portugal programme for financial support through the project 0377_Iberphenol_6_E; the European Regional Development Fund (ERDF) to FEDER through the Regional Operational Program North 2020, within the scope of Project NORTE-01-0145-FEDER-023289: DeCodE and project Mobilizador Norte-01-0247-FEDER-024479: ValorNatural $^{\circledast}$.

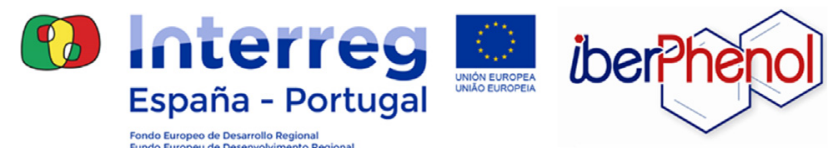




\section{Appendix A. Supplementary material}

Supplementary data to this article can be found online at https:// doi.org/10.1016/j.jff.2019.02.037.

\section{References}

Abreu, R. M., Ferreira, I. C., Calhelha, R. C., Lima, R. T., Vasconcelos, M. H., Adega, F., .. Queiroz, M. J. R. (2011). Anti-hepatocellular carcinoma activity using human HepG2 cells and hepatotoxicity of 6 -substituted methyl 3-aminothieno [3, 2-b] pyridine-2carboxylate derivatives: In vitro evaluation, cell cycle analysis and QSAR studies. European Journal of Medicinal Chemistry, 46, 5800-5806.

AOAC International, \& George, W., Jr. (2016). Association of Official Analytical Chemists (20th ed.). Rockville, MD, USA: AOAC.

Banerjee, K., Banerjee, S., Das, S., \& Mandal, M. (2015). Probing the potential of apigenin liposomes in enhancing bacterial membrane perturbation and integrity loss. Journal of Colloid and Interface Science, 453, 48-59.

Bessada, S. M., Barreira, J. C. M., Barros, L., Ferreira, I. C. F. R., \& Oliveira, M. B. P. P. (2016). Phenolic profile and antioxidant activity of Coleostephus myconis (L.) Rchb. f.: An underexploited and highly disseminated species. Industrial Crops and Products, 89, 45-51.

Bicudo, M. O. P., Ribani, R. H., \& Beta, T. (2014). Anthocyanins, phenolic acids and antioxidant properties of juçara fruits (Euterpe edulis M.) along the on-tree ripening process. Plant Foods for Human Nutrition, 69, 142-147.

Borges, G. D. S. C., Gonzaga, L. V., Jardini, F. A., Mancini Filho, J., Heller, M., Micke, G., ... Fett, R. (2013). Protective effect of Euterpe edulis M. on Vero cell culture and antioxidant evaluation based on phenolic composition using HPLC-ESI-MS/MS. Food Research International, 51, 363-369.

Cardoso, A. L., Di Pietro, P. F., Vieira, F. G. K., Boaventura, B. C. B., de Liz, S., Borges, G. D. S. C., ... da Silva, E. L. (2015). Acute consumption of juçara juice (Euterpe edulis) and antioxidant activity in healthy individuals. Journal of Functional Foods, 17, $152-162$.

Corrêa, R. C., Barros, L., Fernandes, Â., Sokovic, M., Bracht, A., Peralta, R. M., \& Ferreira, I. C. (2018). A natural food ingredient based on ergosterol: Optimization of the extraction from Agaricus blazei, evaluation of bioactive properties and incorporation in yogurts. Food \& Function, 9, 1465-1474.

Corrêa, R. C. G., de Souza, A. H. P., Calhelha, R. C., Barros, L., Glamoclija, J., Sokovic, M., ... Ferreira, I. C. (2015). Bioactive formulations prepared from fruiting bodies and submerged culture mycelia of the Brazilian edible mushroom Pleurotus ostreatoroseus Singer. Food \& Function, 6, 2155-2164.

del Pilar Garcia-Mendoza, M., Espinosa-Pardo, F. A., Baseggio, A. M., Barbero, G. F., Junior, M. R. M., Rostagno, M. A., \& Martínez, J. (2017). Extraction of phenolic compounds and anthocyanins from juçara (Euterpe edulis Mart.) residues using pressurized liquids and supercritical fluids. The Journal of Supercritical Fluids, 119, 9-16.

Dias, M. I., Barros, L., Morales, P., Cámara, M., Alves, M. J., Oliveira, M. B. P., ... Ferreira, I. C. (2016). Wild Fragaria vesca L. fruits: A rich source of bioactive phytochemicals. Food \& Function, 7, 4523-4532.

Evans, B. C., Nelson, C. E., Shann, S. Y., Beavers, K. R., Kim, A. J., Li, H., ... Duvall, C. L. (2013). Ex vivo red blood cell hemolysis assay for the evaluation of $\mathrm{pH}$-responsive endosomolytic agents for cytosolic delivery of biomacromolecular drugs. Journal of Visualized Experiments: JoVE, e50166.

Favaro, L. I., Balcão, V. M., Rocha, L. K., Silva, E. C., Oliveira, J. M., Jr, Vila, M. M., \& Tubino, M. (2018). Physicochemical characterization of a crude anthocyanin extract from the fruits of jussara (Euterpe edulis Martius): Potential for food and pharmaceutical applications. Journal of the Brazilian Chemical Society, 29, 2072-2088.

Felzenszwalb, I., da Costa Marques, M. R., Mazzei, J. L., \& Aiub, C. A. (2013). Toxicological evaluation of Euterpe edulis: A potential superfruit to be considered. Food and Chemical Toxicology, 58, 536-544.

Ferreres, F., Gonçalves, R. F., Gil-Izquierdo, A., Valentão, P., Silva, A. M., Silva, J. B., ... Andrade, P. B. (2012). Further knowledge on the phenolic profile of Colocasia esculenta (L.) Shott. Journal of Agricultural and Food Chemistry, 60, 7005-7015.

Freitas, R. B., Novaes, R. D., Gonçalves, R. V., Mendonça, B. G., Santos, E. C., Ribeiro, A. Q., ... Leite, J. P. V. (2016). Euterpe edulis extract but not oil enhances antioxidant defenses and protects against nonalcoholic fatty liver disease induced by a high-fat diet in rats. Oxidative Medicine and Cellular Longevity Article ID 8173876.

Geraldi, M. V., Tulini, F. L., Souza, V. M., \& De Martinis, E. C. (2018). Development of yoghurt with juçara pulp (Euterpe edulis M.) and the probiotic Lactobacillus acidophilus La5. Probiotics and Antimicrobial Proteins, 10, 71-76.

Gonçalves, G. A., Soares, A. A., Correa, R. C., Barros, L., Haminiuk, C. W., Peralta, R. M., ... Bracht, A. (2017). Merlot grape pomace hydroalcoholic extract improves the oxidative and inflammatory states of rats with adjuvant-induced arthritis. Journal of Functional Foods, 33, 408-418.

Guergoletto, K. B., Mauro, C. S. I., \& Garcia, S. (2017). Juçara (Euterpe edulis) pulp as a substrate for probiotic bacteria fermentation: Optimisation process and antioxidant activity. Emirates Journal of Food and Agriculture, 29, 949-959.

Guergoletto, K. B., Costabile, A., Flores, G., Garcia, S., \& Gibson, G. R. (2016). In vitro fermentation of juçara pulp (Euterpe edulis) by human colonic microbiota. Food Chemistry, 196, 251-258.
Inada, K. O. P., Oliveira, A. A., Revorêdo, T. B., Martins, A. B. N., Lacerda, E. C. Q., Freire, A. S., ... Monteiro, M. C. (2015). Screening of the chemical composition and occurring antioxidants in jabuticaba (Myrciaria jaboticaba) and jussara (Euterpe edulis) fruits and their fractions. Journal of Functional Foods, 17, 422-433.

Khoo, H. E., Azlan, A., Tang, S. T., \& Lim, S. M. (2017). Anthocyanidins and anthocyanins: Colored pigments as food, pharmaceutical ingredients, and the potential health benefits. Food \& Nutrition Research, 61, 1361779.

Koehnlein, E. A., Koehnlein, É. M., Corrêa, R. C. G., Nishida, V. S., Correa, V. G., Bracht, A., \& Peralta, R. M. (2016). Analysis of a whole diet in terms of phenolic content and antioxidant capacity: Effects of a simulated gastrointestinal digestion. International Journal of Food Sciences and Nutrition, 67, 614-623.

Liu, R., Zhang, T., Yang, H., Lan, X., Ying, J., \& Du, G. (2011). The flavonoid apigenin protects brain neurovascular coupling against amyloid- $\beta$ 25-35-induced toxicity in mice. Journal of Alzheimer's Disease, 24, 85-100.

Madunić, J., Madunić, I. V., Gajski, G., Popić, J., \& Garaj-Vrhovac, V. (2018). Apigenin: A dietary flavonoid with diverse anticancer properties. Cancer Letters, 413, 11-22.

Pereira, E., Barros, L., Calhelha, R. C., Duenas, M., Carvalho, A. M., Santos-Buelga, C., \& Ferreira, I. C. (2014). Bioactivity and phytochemical characterization of Arenaria montana L. Food \& Function, 5(8), 1848-1855.

Pérez-Sánchez, A., Sánchez-Marzo, N., Barrajón-Catalán, E., Castillo, J., Herranz-López, M., \& Micol, V. (2017). Antioxidant activity and intestinal absorption of apigenin and its potassium salt derivative in Caco-2 cell monolayers: P-179. Free Radical Biology and Medicine, 108, S79.

Petroni, K., Trinei, M., Fornari, M., Calvenzani, V., Marinelli, A., Micheli, L. A., ... Giorgio, M. (2017). Dietary cyanidin 3-glucoside from purple corn ameliorates doxorubicininduced cardiotoxicity in mice. Nutrition, Metabolism and Cardiovascular Diseases, 27, 462-469.

Pires, T. C., Dias, M. I., Barros, L., Alves, M. J., Oliveira, M. B. P., Santos-Buelga, C., \& Ferreira, I. C. (2018). Antioxidant and antimicrobial properties of dried Portuguese apple variety (Malus domestica Borkh. cv Bravo de Esmolfe). Food Chemistry, 240, 701-706.

Rai, M., Paralikar, P., Jogee, P., Agarkar, G., Ingle, A. P., Derita, M., \& Zacchino, S. (2017). Synergistic antimicrobial potential of essential oils in combination with nanoparticles: Emerging trends and future perspectives. International Journal of Pharmaceutics, 519, 67-78.

Ribeiro, L. O., Pereira, R. N., Tonon, R. V., Cabral, L. M. C., Santiago, M. C. P., Vicente, A. A., ... Freitas, S. P. (2018). Antioxidant compounds recovery from Juçara residue by thermal assisted extraction. Plant Foods for Human Nutrition, 73, 68-73.

Rodrigues, S., Calhelha, R. C., Barreira, J. C., Dueñas, M., Carvalho, A. M., Abreu, R. M., ... Ferreira, I. C. (2012). Crataegus monogyna buds and fruits phenolic extracts: Growth inhibitory activity on human tumor cell lines and chemical characterization by HPLC-DAD-ESI/MS. Food Research International, 49(1), 516-523.

Rupasinghe, H. V., Arumuggam, N., Amararathna, M., \& De Silva, A. B. K. H. (2018). The potential health benefits of haskap (Lonicera caerulea L.): Role of cyanidin-3-O-glucoside. Journal of Functional Foods, 44, 24-39.

Sánchez-Rangel, J. C., Benavides, J., Heredia, J. B., Cisneros-Zevallos, L., \& JacoboVelázquez, D. A. (2013). The Folin-Ciocalteu assay revisited: Improvement of its specificity for total phenolic content determination. Analytical Methods, 5, 5990-5999.

Schulz, M., Biluca, F. C., Gonzaga, L. V., Borges, G. D. S. C., Vitali, L., Micke, G. A., ... Costa, A. C. O. (2017). Bioaccessibility of bioactive compounds and antioxidant potential of juçara fruits (Euterpe edulis Martius) subjected to in vitro gastrointestinal digestion. Food Chemistry, 228, 447-454.

Schulz, M., Borges, G. D. S. C., Gonzaga, L. V., Costa, A. C. O., \& Fett, R. (2016). Juçara fruit (Euterpe edulis Mart.): Sustainable exploitation of a source of bioactive compounds. Food Research International, 89, 14-26.

Schulz, M., Borges, G. D. S. C., Gonzaga, L. V., Seraglio, S. K. T., Olivo, I. S., Azevedo, M. S., ... Spudeit, D. A. (2015). Chemical composition, bioactive compounds and antioxidant capacity of juçara fruit (Euterpe edulis Martius) during ripening. Food Research International, 77, 125-131.

Shelef, O., Weisberg, P. J., \& Provenza, F. D. (2017). The value of native plants and local production in an era of global agriculture. Frontiers in Plant Science, 8, 2069.

Silva, N. A. D., Rodrigues, E., Mercadante, A. Z., \& de Rosso, V. V. (2014). Phenolic compounds and carotenoids from four fruits native from the Brazilian Atlantic forest. Journal of Agricultural and Food Chemistry, 62, 5072-5084.

Takebayashi, J., Iwahashi, N., Ishimi, Y., \& Tai, A. (2012). Development of a simple 96well plate method for evaluation of antioxidant activity based on the oxidative haemolysis inhibition assay (OxHLIA). Food Chemistry, 134, 606-610.

Vieira, G. S., Marques, A. S., Machado, M. T., Silva, V. M., \& Hubinger, M. D. (2017). Determination of anthocyanins and non-anthocyanin polyphenols by ultra performance liquid chromatography/electrospray ionization mass spectrometry (UPLC/ ESI-MS) in jussara (Euterpe edulis) extracts. Journal of Food Science and Technology, 54, 2135-2144.

Vieira, G. S., Moreira, F. K., Matsumoto, R. L., Michelon, M., Francisco Filho, M., \& Hubinger, M. D. (2018). Influence of nanofiltration membrane features on enrichment of jussara ethanolic extract (Euterpe edulis) in anthocyanins. Journal of Food Engineering, 226, 31-41.

Wang, F., Zhou, R. J., Zhao, X., Ye, H., \& Xie, M. L. (2018). Apigenin inhibits ethanolinduced oxidative stress and LPS-induced inflammatory cytokine production in cultured rat hepatocytes. Journal of Applied Biomedicine, 16(1), 75-80. 